\title{
OSCILLATORY FLOW NEAR A STAGNATION POINT*
}

\author{
M. G. BLYTH ${ }^{\dagger}$ AND P. HALL ${ }^{\ddagger}$
}

\begin{abstract}
The classical Hiemenz solution describes incompressible two-dimensional stagnation point flow at a solid wall. We consider an unsteady version of this problem, examining particularly the response close to the wall when the solution at infinity is modulated in time by a periodic factor of specified amplitude and frequency. While this problem has already been tackled in the literature for general frequency in cases when the amplitude of the time-periodic factor is either large or small, we compute the flow for arbitrary values of both these parameters. For any given amplitude, we find that there exists a threshold frequency above which the flow is regular and periodic, with the same period as the modulation factor, and beneath which the solution terminates in a finite time singularity. The dividing line in parameter space between these two possibilities is identified and favorably compared with the predictions of asymptotic analyses in the small and large frequency limits.
\end{abstract}

Key words. stagnation point, boundary layer, steady streaming

AMS subject classification. 76D10

DOI. $10.1137 /$ S0036139902408175

1. Introduction. The classical Hiemenz solution describes the flow near a stagnation point on a plane wall and may be found in Batchelor [1]. When this flow is modulated in time by a periodic multiplicative factor on the streamfunction at infinity, the resulting solution can be used to describe the local dynamics around a stagnation point on an oscillating body. Equally, the body may be thought of as fixed, with the flow out in the far field varying periodically in time. The steady streaming motion established by the Reynolds stresses associated with the oscillatory motion (Stuart [2]) is an important feature of such time-periodic flows. This effect occurs, for example, around a transversely oscillating circular cylinder (Schlichting [3]). When the amplitude of the far field fluctuation is small, modified Hiemenz flow can be used to model the local effects of disturbances such as acoustic noise impinging on the boundary layer around a translating bluff body. More generally, such problems fall within the purview of receptivity theory (e.g., Erturk and Corke [4], Morkovin [5]). We are concerned with the behavior in the vicinity of the stagnation point, where the body surface may be considered to be locally flat. In this context we allow for fluctuations of arbitrary amplitude and frequency.

Other studies pertinent to this modified Hiemenz problem include those by Grosch and Salwen [6] and Merchant and Davis [7]; among earlier investigations, we mention those by Matunobu [8], Pedley [9], and Ishigaki [10]; see also Lighthill [11]. Grosch and Salwen, while confining their attention to small free stream fluctuations, examined the flow when the frequency of these disturbances is either small or large. Expanding in power series in the disturbance amplitude and Fourier series in time, they showed that to leading order the low frequency case is merely a quasi-steady version of the classical Hiemenz solution, while the high frequency case exhibits a double boundary layer structure similar to that first discussed by Riley [12] and Stuart [2] for oscillating

${ }^{*}$ Received by the editors May 20, 2002; accepted for publication (in revised form) December 3, 2002; published electronically June 12, 2003.

http://www.siam.org/journals/siap/63-5/40817.html

${ }^{\dagger}$ School of Mathematics, University of East Anglia, Norwich, UK, NR4 7TJ (m.blyth@uea.ac.uk).

${ }^{\ddagger}$ Mathematics Department, Imperial College, 180 Queen’s Gate, London, UK, SW7 2BZ (philhall@ic.ac.uk). 
flows. Merchant and Davis tackled the same unsteady Hiemenz problem, but also examined the flow structure when the mean component of the free stream is very much smaller than the oscillatory part. In this case a double boundary layer structure is once again revealed, although the authors showed that no solutions exist when the mean component of the free stream drops below a certain cut-off point. This is not to say that solutions with a different asymptotic form do not exist at smaller values of the free stream mean. We address this point in the current work.

Our interest in the problem was prompted by Hall and Papageorgiou's [13] recent study of unsteady incompressible flow induced in an infinite channel when the walls pulsate uniformly in space and periodically in time. Assuming a stagnation point structure for this flow, they demonstrated numerically the existence of purely periodic, quasi-periodic, and even chaotic flow solutions, depending on the frequency and amplitude of the wall motion. Our aim was to investigate the possibility of such varied dynamics for a periodically forced stagnation point flow in a semi-infinite domain. In fact, numerical solution of the unsteady Hiemenz problem shows that for many parameter values a singularity is encountered at a finite time. This eventuality is perhaps unsurprising in light of previous studies of colliding boundary layers or those in which the external flow reverses direction, where such singularities may also be found. One example is the flow over a rotating disk in a counter-rotating fluid, whose near-singularity structure was described by [14]. Another study of particular relevance to the current work is that by Riley and Vasantha [15], who considered the same problem as ours but with a purely oscillatory (zero mean) free stream. In this case the equations break down in finite time for any value of the forcing frequency. The breakdown was interpreted by Riley and Vasantha as the result of drifting fluid particles in the steady streaming layer accumulating at the stagnation point and ultimately causing an eruption of fluid from the boundary layer. The nature of the finite time singularity was found to have the same form as that occurring near the equator of an impulsively started sphere, as studied by Banks and Zaturska [16]. When the flow in the far field has nonzero mean, as is to be discussed here, the near-singularity structure is also described by Banks and Zaturska's analysis. The blow-up is not localized in space but occurs over the entire flow domain.

That the flow can break down when the mean component of the free stream is nonzero is not mentioned by either Grosch and Salwen or Merchant and Davis. However, the former authors demonstrate that the inclusion of a nonzero mean component in the free stream can allow the solution to be continued indefinitely without breakdown. We have found that this is true, provided that a condition between the fluctuation amplitude and frequency is not violated. The condition amounts to a threshold frequency, at any given amplitude, below which blow-up will occur but above which the solution remains regular. It is still conceivable that aperiodic or even chaotic solutions might exist in the large frequency limit, so long as this condition is satisfied. However, we have not been able to identify any such solutions despite extensive numerical searches. At all candidate parameter values tested, the solutions remain periodic with frequency equal to that of the free stream fluctuation. Nevertheless, the nature of the condition under which breakdown occurs is of interest, and in this sense our work constitutes a worthwhile extension of the previous studies.

We begin with a problem description, followed by a brief discussion of the numerical methods utilized to solve the governing equations. Results are then presented together with asymptotic analyses in the small and large frequency limits, and comparison is made between the two. We conclude with a short note on the axisymmetric version of the problem. 
2. Problem statement and numerical method. The problem under consideration is that of two-dimensional unsteady Hiemenz flow approaching a flat plate. Referring to a set of Cartesian axes $(x, y)$, the flat plate occupies $-\infty<x<\infty$, $y=0$. We define velocity components $U_{\infty} u(x, y, t), U_{\infty} v(x, y, t)$ in the $x, y$ directions, respectively, where $U_{\infty}$ is the typical flow speed in the far field. In this region the flow is potential, with $u=(x / l) U(t), v=-(y / l) U(t)$, where

$$
U(t)=1+\Delta \cos (\omega t)
$$

for a chosen amplitude $\Delta$ and frequency $\omega$. The remaining parameters are the kinematic viscosity $\nu$ and an arbitrarily chosen length scale $l$.

As in the steady case, it is reasonable to assume that the same simple velocity dependence on the $x$ coordinate also applies in the viscous layer close to the plate. In this region we introduce the new coordinate $\eta=(y / l) R^{1 / 2}$ and set

$$
u=\left(\frac{x}{l}\right) F_{\eta}(\eta, \tau), \quad v=-R^{-1 / 2} F(\eta, \tau)
$$

defining the Reynolds number to be $R=U_{\infty} l / \nu$ and introducing the new time variable $\tau=\omega t$. Note that we do not require $R$ to be large. Finally, defining the Strouhal number

$$
\sigma=\frac{\omega l}{U_{\infty}}
$$

and setting $a(\tau)=1+\Delta \cos \tau$, we may express the wall layer system as

$$
\sigma F_{\eta \tau}+F_{\eta}^{2}-F F_{\eta \eta}=\sigma a_{\tau}+a^{2}+F_{\eta \eta \eta}
$$

with

$$
F(0, \tau)=F_{\eta}(0, \tau)=0, \quad F_{\eta} \rightarrow a(\tau) \quad \text { as } \quad \eta \rightarrow \infty,
$$

to satisfy the solid wall boundary conditions and to match to the outer potential solution. When $\Delta=0$, the problem reduces to that of classical steady Hiemenz flow. The temporally periodic part of (2.1) represents a superimposed disturbance on the steady far field solution. The response to this disturbance close to the plate is quantified by solving (2.2) and (2.3) for different values of the fluctuation amplitude $\Delta$ and the Strouhal number $\sigma$. We emphasize that $\Delta$ is not restricted to being small.

At this stage we note that functions satisfying (2.2) and (2.3) represent exact solutions of the Navier-Stokes equations since no approximations have been made. In addition, while the Reynolds number is not required to be large in this analysis, for ease of description we shall refer to the main flow governed by (2.2) as the Hiemenz boundary layer, even though no conventional boundary layer approximation is necessary.

Asymptotic solutions are possible in the limits of small and large frequency, and these will be discussed in a later section. For general values of the parameters $(\Delta, \sigma)$ numerical methods must be used to solve the wall layer equations. To expedite the numerical solution, we introduce the function $G=F_{\eta}$ and write the equations in the form

$$
\begin{aligned}
\sigma G_{\tau}+G^{2}-F G_{\eta} & =\sigma a_{\tau}+a^{2}+G_{\eta \eta}, \\
G & =F_{\eta},
\end{aligned}
$$


with

$$
F(0, \tau)=G(0, \tau)=0, \quad G \rightarrow a(\tau) \quad \text { as } \quad \eta \rightarrow \infty .
$$

We found that calculations should be started at $\tau=\pi / 2$ rather than $\tau=0$; otherwise, when $\Delta \gg 1$, the forcing is too large when the integration is initiated and the numerical solution blows up instantaneously. In practice, the initial profile makes negligible difference to the computation of the singular time, and calculations were always begun with $F=G=0$. To march forwards in time we used either the second order accurate Crank-Nicholson method or the fourth order accurate Runge-Kutta integration, both with a second order accurate spatial discretization. Thus some independent numerical check on our results was available. Most of the results presented in this paper were computed using the latter of the two schemes. However, at various stages the computations were repeated using the Crank-Nicholson method, and these always provided good agreement. For large $\sigma$, we found it useful to introduce a stretched grid in order to insert many more points in the Stokes shear-wave layer next to the wall, where the most significant changes in the flow are concentrated. We present some of our results in the next section.

3. Analytical discussion and results. Grosch and Salwen [6] show that, for sufficiently small amplitude and in the limit of vanishing Strouhal number, the quasisteady Hiemenz solution describes the flow to leading order. Specifically, to $O(\sigma)$, the solution may be written as $F=a(\tau)^{1 / 2} f\left(a^{1 / 2} \eta\right)$, where $f$ satisfies the usual steady Hiemenz equation and boundary conditions. When $\sigma \rightarrow \infty$, again for small enough $\Delta$, the solution adopts a double boundary layer structure, similar to that analyzed by Riley [12] and Stuart [2]. In this case the boundary layer splits into two regions. In the lower region, the Stokes layer, the solution is periodic to leading order. The nonlinear terms in the equations generate a small steady component, which persists to the upper reaches of the Stokes layer and acts to drive a steady streaming flow in the outer part of the Hiemenz boundary layer. For both small and large $\sigma$, Grosch and Salwen expanded in Taylor series in the amplitude $\Delta$ and Fourier series in time. While they were not able to determine the radius of convergence of their series exactly, they estimated that for large $\sigma$ the series should converge when $\Delta<\sigma$. Later Merchant and Davis [7] showed that if both the amplitude and the Strouhal number are large, and if the thickness of the main boundary layer and the induced steady-streaming layer are chosen to coincide, then no solutions exist in our notation when $\Delta>1.289 \sigma^{1 / 2}$. This derives from the fact that the leading order steady-streaming equation has no solution when the amplitude exceeds this bound. However, this does not deny the existence of other solutions with a different boundary layer structure in these limits. In the Merchant and Davis flow structure the Stokes layer is linear to leading order. If instead we hypothesize that solutions exist wherein the nonlinear terms are of the same order of magnitude as the unsteady terms, corresponding to the scaling $\Delta \sim \sigma$ as $\sigma \rightarrow \infty$, we find that the equations for the leading order Stokes layer problem, with the appropriate matching condition at infinity, are the same as those studied by Riley and Vasantha [15]. These correspond to (2.2) and (2.3) with $a(\tau)=\cos \tau$. Riley and Vasantha's results show that these slightly reduced equations terminate in a singularity at a finite time for all values of $\sigma$. In due course we shall present numerical evidence that no regular solutions exist above the limit laid down by Merchant and Davis.

As a preliminary test of our codes, we computed small amplitude solutions and obtained results in excellent agreement with those of Grosch and Salwen. For large $\sigma$, 


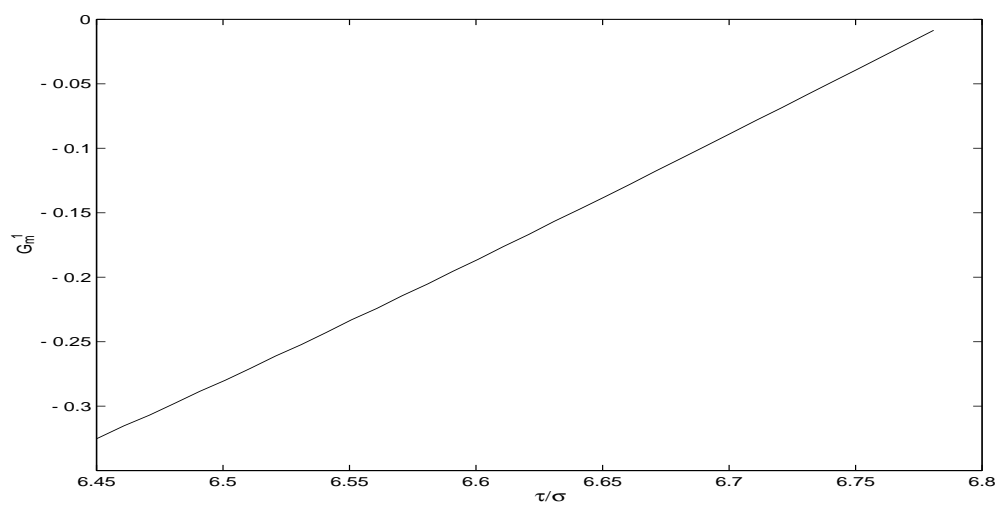

FIG. 3.1. $\Delta=2.0, \sigma=0.5$ : The locus of $G_{m}^{-1}=1 / \min _{\eta}\{G\}$ in time, $\tau$, close to the singularity at $\tau_{s}=3.39$.

and for $\Delta$ of typical size $O\left(\sigma^{1 / 2}\right)$, we compared our results with the first two terms in the asymptotic expansion of Merchant and Davis and again found that our numerics were in excellent agreement with the theory. For a fixed $\sigma$, however, we discovered that when the value of $\Delta$ exceeds a certain limit, the integration blows up at a finite time singularity. We shall henceforth label the singular time $\tau_{s}$. The flow structure in the vicinity of $\tau_{s}$ is the same as that arising near the equator of an impulsively started sphere, a problem analyzed by Banks and Zaturska [16]. As the singularity is approached in that situation, the most important terms in the near-equator equations over the main part of the flow combine to mimic those of our own equation. Close to the singular time the flow acquires a three-tiered structure, with viscous zones at the wall and infinity sandwiching an inviscid core region. In this sense the flow is similar to that attained just prior to the breakdown which occurs a short time after the direction of a rotating disk is suddenly reversed. The near-singularity structure for this flow has been examined by Stewartson and Bodonyi [17] and corrected by Stewartson, Simpson, and Bodonyi [14]. (See also Ockendon [18] for a discussion of a steady rotating disk flow with a similar three-zone structure.) While Banks and Zaturska do not give the details of the flow in the upper and lower viscous regions, we have confirmed that the arguments of Stewartson, Simpson, and Bodonyi may be adapted accordingly. Further discussion of the viscous zones is suppressed. Instead we demonstrate that the flow behavior in the middle region is consistent with that of Banks and Zaturska. By comparison with their theory, in the main part of the flow we expect $\eta$ to scale like $\left(\tau_{s}-\tau\right)^{-1 / 2}$, and thus we write $\widehat{\eta}=\eta\left(\tau_{s}-\tau\right)^{1 / 2}$. From their predictions we anticipate that $F \propto\left(\tau_{s}-\tau\right)^{-3 / 2} \phi(\widehat{\eta})$, and thus $G \propto\left(\tau_{s}-\tau\right)^{-1}$ as $\tau \rightarrow \tau_{s}^{-}$. As time progresses we observe that, at a given $\tau$, the $G(\eta, \tau)$ profile has at most one local minimum $G_{m}$ at $\eta=\eta_{m}$. Tracking the inverse of this minimum value up to the singular time for the case $\Delta=2.0, \sigma=0.5$, we plot the graph shown in Figure 3.1. The relationship between $G_{m}^{-1}$ and $\tau$ appears convincingly linear. Assuming this to be the case, a more accurate value for $\tau_{s}$ may be predicted by means of linear interpolation. Similarly, plotting $\eta_{m}^{-2}$ against $\tau$ close to the singular time reveals an apparent linear dependence which is equally compelling. The critical point dividing singular and periodic solutions at this frequency is $\Delta \approx 1.537$. As the amplitude approaches this value from above, we find that the singular time $\tau_{s}$ increases without bound. 

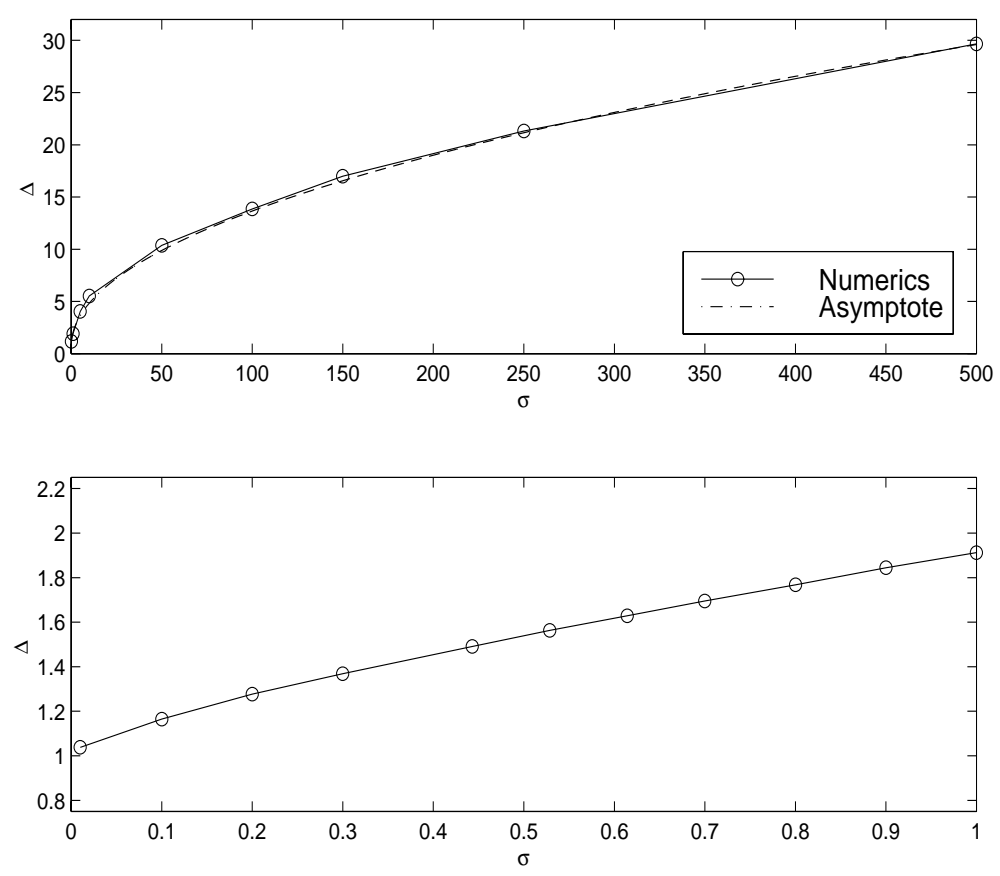

FIG. 3.2. The barrier in $(\Delta, \sigma)$ parameter space between regular periodic solutions (below the line) and those which blow up in finite time (above the line). The lower graph shows the barrier close to $\sigma=0$. The upper graph includes the asymptotic approximation $\Delta \sim 1.289 \sigma^{1 / 2}+0.76$ for large $\sigma$ (see section 3.1 ).

We now turn to the general picture in $(\Delta, \sigma)$ parameter space. Using the numerical procedure described above, we have identified the parametric regions in which the solutions either remain regular and periodic or else become singular in finite time. The parameter space is divided neatly into two regions by the curve depicted in Figure 3.2. We believe that points on this curve have been calculated accurately to within $0.1 \%$. Flows corresponding to points $(\Delta, \sigma)$ lying below the curve are periodic, while those lying above eventually reach a singularity. We have calculated solutions up to and including $\sigma=1000$ and, for the regular solutions, have encountered no bifurcations leading to aperiodic flows. Rather, the solutions remain periodic with the same period as that of the far field disturbance.

Also shown in Figure 3.2 is the asymptotic approximation valid for large values of $\sigma$, to be discussed shortly. Magnifying the curve in the region of zero frequency shows that it approaches unity as $\sigma \rightarrow 0$, suggesting that, for temporally slowly varying flows of this kind, only a small amount of flow reversal can be tolerated if the solution is to remain regular. This limit is discussed in due course.

When the disturbance frequency is large, the boundary layer supports a small steady flow component, driven by a residual slip velocity from a Stokes layer beneath. The analysis of Merchant and Davis suggests that no regular solutions exist when the amplitude is also large, specifically when $\Delta>1.289 \sigma^{1 / 2}$, since then the system governing the steady streaming component has no solution. In order to provide the best agreement between our numerical calculations and the large amplitude theory, we have found it worthwhile to compute the next term in this asymptotic expansion. 
In what follows, we therefore supply a brief description of the asymptotic flow at large frequencies.

3.1. Large amplitude and frequency. It is convenient at this stage to rescale (2.2), (2.3) by writing $\eta=\Delta^{-1 / 2} \widehat{\eta}, F=\Delta^{-1 / 2} \widehat{F}, \sigma=\Delta \Omega$ and introducing the small parameter $\varepsilon=1 / \Delta$. This leads to the equivalent system:

$$
\Omega \widehat{F}_{\widehat{\eta} \tau}+\widehat{F}_{\hat{\eta}}^{2}-\widehat{F} \widehat{F}_{\widehat{\eta} \hat{\eta}}=-\Omega \sin \tau+(\varepsilon+\cos \tau)^{2}+\widehat{F}_{\widehat{\eta} \hat{\eta} \hat{\eta}},
$$

with

$$
\widehat{F}(0, \tau)=\widehat{F}_{\widehat{\eta}}(0, \tau)=0, \quad \widehat{F}_{\widehat{\eta}} \rightarrow \varepsilon+\cos \tau \quad \text { as } \quad \widehat{\eta} \rightarrow \infty .
$$

Now, when $\varepsilon$ is small and $\Omega$ is large, we look for solutions with $\varepsilon=a_{0} \Omega^{-1}+$ $a_{1} \Omega^{-2}+\cdots$. In the Stokes layer, of thickness $O\left(\Omega^{-1 / 2}\right)$, the expansion proceeds as

$$
\widehat{F}=\Omega^{-1 / 2} \phi_{0}(\xi, \tau)+\Omega^{-3 / 2} \phi_{1}(\xi, \tau)+\cdots,
$$

where $\xi=\Omega^{1 / 2} \widehat{\eta}$ is a scaled coordinate normal to the wall. The first order solution is given by

$$
\phi_{0}(\xi, \tau)=\xi \cos \tau-\cos \left(\tau-\frac{\pi}{4}\right)+\mathrm{e}^{-\xi / \sqrt{2}} \cos \left(\tau-\frac{\xi}{\sqrt{2}}-\frac{\pi}{4}\right) .
$$

At second order, the solution may be written as $\phi_{1}(\xi, \tau)=\frac{1}{2} \phi_{M}(\xi, \tau)$, where $\phi_{M}$ is a somewhat lengthy expression appearing as formula (3.20c) of Merchant and Davis's paper. As pointed out by Stuart [2], it is not possible to satisfy the infinity condition at this order; rather a steady slip velocity persists at the top of the Stokes layer, driving a steady streaming motion above. Therefore we simply note at this stage that $\phi_{1 \xi}(\infty, \tau)=-3 / 4$.

With the current choice of scaling, the streaming layer has the same thickness as the Hiemenz boundary layer (of order $\Omega^{1 / 2}$ in this notation). Introducing the new coordinate $\zeta=\Omega^{-1 / 2} \widehat{\eta}$, the relevant expansion is

$$
\widehat{G}=\Omega^{-1 / 2}\left\{\psi_{0}(\zeta, \tau)+f_{0}(\zeta)\right\}+\Omega^{-3 / 2}\left\{\psi_{1}(\zeta, \tau)+f_{1}(\zeta)\right\}+\cdots,
$$

where $\widehat{G}=\widehat{F}-\Omega^{1 / 2} \zeta \cos (\tau)-\Omega^{-1 / 2} \cos (\tau-\pi / 4)$. The functions $\psi_{i}$ equal zero when averaged over a single time period. In fact $\psi_{0} \equiv 0$, and the first order streaming problem is given by

$$
f_{0}^{\prime \prime \prime}+f_{0} f_{0}^{\prime \prime}-f_{0}^{\prime 2}+a_{0}^{2}=0
$$

with

$$
f_{0}(0)=0, \quad f_{0}^{\prime}(0)=\frac{-3}{4}, \quad f_{0}^{\prime}(\infty)=a_{0} .
$$

A numerical treatment of this problem by both Merchant and Davis and also by Riley and Weidman [19] indicates that a unique solution exists when $0<a_{0}<3 / 4$, two solutions exist when $3 / 4<a_{0}<a_{0 c}$, and no solutions exist when $a_{0}>a_{0 c}$, where $a_{0 c} \approx 0.602$. Continuing, we derive the second order streaming problem:

$$
f_{1}^{\prime \prime \prime}+f_{0} f_{1}^{\prime \prime}-2 f_{0}^{\prime} f_{1}^{\prime}+f_{0}^{\prime \prime} f_{1}=\frac{1}{2 \sqrt{2}} f_{0}^{\prime \prime}-2 a_{0} a_{1},
$$


with

$$
f_{1}(0)=\frac{13}{4 \sqrt{2}}, \quad f_{1}^{\prime}(0)=0, \quad f_{1}^{\prime}(\infty)=a_{1} .
$$

Taking $a_{1}=0$ should reduce the problem to the corresponding equation of Merchant and Davis. However, we remark that this leaves a right-hand side proportional to $f_{0}^{\prime \prime}$, which is absent in that paper and which we believe should be included.

Our concern now is to calculate $a_{1}$ when $a_{0}=a_{0 c}$. Numerical trials suggest that a solution exists for the value $a_{1}=-0.55$ and is unique. Reverting to our original notation, the asymptotic approximation for the critical amplitude proceeds as

$$
\Delta=1.29 \sigma^{1 / 2}+0.76+O\left(\sigma^{-1 / 2}\right) \quad \text { as } \quad \sigma \rightarrow \infty .
$$

This is plotted in Figure 3.2 along with our full numerical results. It provides strong evidence that it is this asymptote which defines the barrier between large frequency solutions remaining regular and periodic and those encountering a finite time singularity.

3.2. Small frequency. We now focus our attention on small frequency solutions. The numerical calculations suggest that the barrier between regular and singular solutions approaches $\Delta=1$ as $\sigma \rightarrow 0$ (see Figure 3.2). Naturally, when $\sigma=0$ we obtain classical Hiemenz flow and therefore expect steady solutions at any amplitude. In this sense we expect $\sigma \rightarrow 0$ to be a singular limit. While considering a similar flow but with zero mean at infinity, Riley and Vasantha [15] showed that the singular time grows without bound as the fluctuation frequency tends to zero. Paralleling their analysis, we now examine the solution close to $\sigma=0$.

When both the frequency and amplitude are small, the flow follows the quasisteady solution given by Grosch and Salwen [6] and mentioned above in section 3. However, if the amplitude equals or exceeds unity, $a(\tau)$ has a zero at $\tau=\tau_{0}$, where $\tau_{0}=\pi-\cos ^{-1}(1 / \Delta)$. In what follows, $\Delta-1$ is assumed to be nonnegative (but not necessarily small) so that $a(\tau)$ has such a zero. The quasi-steady approximation will break down when $\tau$ approaches $\tau_{0}$, as the unsteady terms, which were hitherto small, grow to become comparable in size with the others. A consideration of the relative magnitudes of terms in (2.2) suggests that the quasi-steady approximation will become invalid when $a(\tau)=O\left(\sigma^{1 / 2}\right)$.

In the vicinity of $\tau=\tau_{0}$, a balance of the terms in (2.2) suggests the scalings

$$
\left(\tau_{0}-\tau\right)=\sigma^{1 / 2} T, \quad \eta=\sigma^{-1 / 4} Y, \quad F=\sigma^{1 / 4} \widetilde{F}(Y, T)
$$

for new order one variables $T, Y, \widetilde{F}$. Neglecting terms of order $o\left(\sigma^{4 / 3}\right)$, the governing system reduces to

$$
\begin{array}{r}
-\widetilde{F}_{Y T}+\widetilde{F}_{Y}^{2}-\widetilde{F} \widetilde{F}_{Y Y}=-\mu+\mu^{2} T^{2}+\widetilde{F}_{Y Y Y}, \\
\widetilde{F}(0, T)=\widetilde{F}_{Y}(0, T)=0, \quad \widetilde{F}_{Y} \rightarrow \mu T \text { as } \quad Y \rightarrow \infty,
\end{array}
$$

where $\mu=(\Delta-1)^{1 / 2}$. As $T \rightarrow \infty, \widetilde{F}$ must match to the quasi-steady Hiemenz solution. We initiate the calculation at $T=T_{\infty}$, where $T_{\infty}$ is sufficiently large, with the profile

$$
\widetilde{F}=\left(\frac{\mu T}{T_{\infty}}\right)^{1 / 2} f(x), \quad \text { with } \quad x=\left(\frac{\mu T}{T_{\infty}}\right)^{1 / 2} Y,
$$



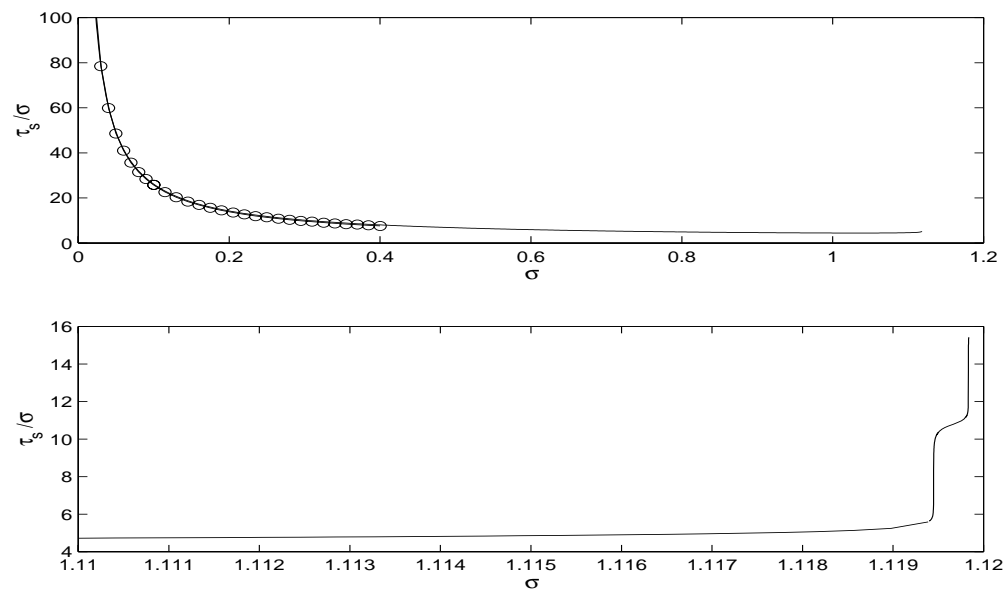

FIG. 3.3. Variation of $\tau_{s}$ with $\sigma$ when $\Delta=2.0$. Top: $\tau_{s}$ in the singular window $\sigma \in(0,1.12)$, plotted with the approximation $\tau_{s} / \sigma=2 \pi / 3 \sigma+1.51 / \sigma^{1 / 2}$ valid as $\sigma \rightarrow 0$ (shown as circles). Bottom: Magnification of the curve close to $\sigma=1.12$.

where $f(x)$ satisfies a scaled version of the steady Hiemenz equations. The system (3.10) is then integrated backwards in time $T$, using a Crank-Nicholson method. For all cases considered, the integration terminates at a singularity after a finite time. The singular time $T_{s}$ is computed using an interpolation procedure similar to that mentioned above for the full numerics.

For the particular case $\Delta=2.0$, we have computed $T_{s}=-1.51$. Thus we predict that

$$
\frac{\tau_{s}}{\sigma}=\frac{2 \pi}{3 \sigma}+\frac{1.51}{\sigma^{1 / 2}}+\cdots \quad \text { as } \quad \sigma \rightarrow 0 .
$$

In Figure 3.3 we show how this estimate compares with the calculated values of $\tau_{s}$ at small frequency obtained by solving the full system $(2.2),(2.3)$. The agreement is very satisfactory. The flow becomes singular for all frequencies in the window $\sigma \in(0,1.12)$, beyond which we cross the barrier in Figure 3.2 and the solutions become periodic. It is interesting to note that as $\sigma \rightarrow 1.12^{-}$, the curve begins to wiggle, a behavior reminiscent of that seen in Riley and Vasantha's problem.

In summary of this short section, we remark that low frequency solutions can become singular as long as $\Delta \geq 1$. When $\Delta<1$, this is not possible, and the flow is quasi-steady and periodic. These conclusions are in agreement with the picture presented in Figure 3.2, where the barrier between regular and irregular solutions approaches unit fluctuation amplitude as the frequency tends to zero.

4. Axisymmetric stagnation point. As a final note, we remark that behavior similar to that described in the previous sections is encountered at an axisymmetric stagnation point. In this case we envisage flow hitting a flat surface and spreading out radially from the stagnation point in the middle. An exact similarity solution with linear dependence in the radial coordinate may then be sought. If we confine our attention to the case in which there is no azimuthal variation, we find that the governing equation and boundary conditions for this flow are almost exactly the same as those for the two-dimensional case, the only difference arising in the nonlinear term, 
where $-2 F F_{\eta \eta}$ appears instead of $-F F_{\eta \eta}$. By analogy with our preceding work, an amplitude $\Delta$ and Strouhal number $\sigma$ may be defined. Riley [20] has studied this flow in detail when the infinity condition has zero mean, and his results suggest that, in common with the two-dimensional stagnation point looked at by Riley and Vasantha, finite time breakdown occurs for all values of the frequency parameter $\sigma$. The flow with nonzero mean at infinity may also be studied and, as in the two-dimensional case, we find that the flow breaks down at a fixed $\sigma$ as soon as a critical value of $\Delta$ is exceeded. A steady-streaming analysis analogous to that performed above again agrees well with the numerically computed results. Unfortunately we once more find no evidence of aperiodic solutions as $\sigma$ is increased (with $\Delta$ remaining below the critical curve for regular solutions).

5. Concluding remarks. We have investigated unsteady stagnation point flow of a modified Hiemenz type. Previously Grosch and Salwen [6] investigated this problem for small fluctuation amplitudes in the low and high frequency limits. Merchant and Davis [7] also considered the large amplitude, high frequency limits, establishing an asymptotic structure in which the streaming region above the Stokes layer is the same thickness as the Hiemenz boundary layer. We have studied the same flow and, for general parameter values, provided numerical evidence that for all frequencies there exists a threshold value of the amplitude beyond which the flow will break down in finite time. The flow structure in the vicinity of the singularity is the same as that arising near the equator of an impulsively started sphere, reported by Banks and Zaturska [16]. Below the threshold value, the solutions are regular and periodic, with period equal to that of the free stream disturbance; in the limit of small frequency, they correspond to the solutions presented by Grosch and Salwen. We have also conducted asymptotic analyses at small and large frequency to predict the dividing line between singular and periodic solutions in these limits. Both have been successfully compared with the results of numerical simulations.

In an earlier study, Riley and Vasantha [15] showed that the same problem with zero mean flow in the free stream breaks down for all possible frequencies. However, when there exists a small mean flow in the free stream, corresponding to the limit of large disturbance amplitude in our problem, our work shows that the solution becomes singular in finite time only when the frequency is smaller than a given value proportional to the square of the amplitude. All frequencies exceeding this value lead to regular, periodic solutions.

\section{REFERENCES}

[1] G. K. Batchelor, An Introduction to Fluid Dynamics, Cambridge University Press, Cambridge, UK, 1967.

[2] J. T. StuART, Double boundary layers in oscillatory viscous flow, J. Fluid Mech., 24 (1966), pp. 673-687.

[3] H. Schlichting, Berechnung ebener periodischer Grenzschichtströmungen, Phys. Z., 33 (1932), pp. 327-335.

[4] E. ERTuRK AND T. C. CoRke, Boundary layer leading-edge receptivity to sound at incidence angles, J. Fluid Mech., 444 (2001), pp. 383-407.

[5] M. V. MoRKovin, Instability, transition to turbulence and predictability, AGARDograph, 236, 1978.

[6] C. E. Grosch and H. Salwen, Oscillating stagnation point flow, Proc. Roy. Soc. London A, 384 (1982), pp. $175-190$.

[7] G. J. Merchant and S. H. Davis, Modulated stagnation point flow and steady streaming, J. Fluid Mech., 198 (1989), pp. 543-555.

[8] Y. Matunobu, Structure of pulsatile Hiemenz flow and temporal variations of wall shear stress near the stagnation point II, J. Phys. Soc. Japan, 43 (1977), pp. 326-329. 
[9] T. J. Pedley, Two-dimensional boundary layers in a free stream which oscillates without reversing, J. Fluid Mech., 55 (1972), pp. 359-383.

[10] M. Ishigaki, Periodic boundary layer near a two-dimensional stagnation point, J. Fluid Mech., 43 (1970), pp. 477-486.

[11] M. J. LighthilL, The response of laminar skin friction and heat transfer to fluctuations in stream velocity, Proc. Roy. Soc. London A, 224 (1954), pp. 1-23.

[12] N. Riley, Oscillating viscous flows, Mathematika, 12 (1965), pp. 161-175.

[13] P. Hall and D. T. Papageorgiou, The onset of chaos in a class of Navier-Stokes solutions, J. Fluid Mech., 393 (1999), pp. 59-87.

[14] K. Stewartson, C. J. Simpson, and R. J. Bodonyi, The unsteady boundary layer on a rotating disk in a counter-rotating fluid. Part 2, J. Fluid Mech., 121 (1982), pp. 507-515.

[15] N. Riley and R. Vasantha, An unsteady stagnation point flow, Quart. J. Mech. Appl. Math., 42 (1988), pp. 511-521.

[16] W. H. H. BANKS AND M. B. ZATURSKA, The collision of unsteady laminar boundary layers, J. Engrg. Math., 13 (1979), pp. 193-212.

[17] K. Stewartson And R. J. Bodonyi, The unsteady laminar boundary layer on a rotating disk in a counter-rotating fluid., J. Fluid Mech., 79 (1977), pp. 669-688.

[18] H. Ockendon, An asymptotic solution for steady flow above an infinite rotating disc with suction, Quart. J. Mech. Appl. Math., 25 (1972), pp. 291-301.

[19] N. Riley And P. D. Weidman, Multiple solutions of the Falkner-Skan equation for flow past a stretching boundary, SIAM J. Appl. Math., 49 (1989), pp. 1350-1358.

[20] N. RiLeY, Unsteady flow at a stagnation point, J. Fluid Mech., 256 (1993), pp. 487-498. 